\title{
Comparison of total variation algorithms for Electrical Impedance Tomography
}

\author{
Zhou Zhou ${ }^{12}$, Gustavo Sato dos Santos², Thomas Dowrick², James Avery², \\ Zhaolin Sun ${ }^{1}$, Hui $\mathrm{Xu}^{1}$ and David S Holder ${ }^{2}$ \\ ${ }^{1}$ National University of Defense Technology, Changsha, 410073, P. R. China \\ ${ }^{2}$ University College London, London, WC1E 6BT, UK \\ zhou.zhou.13@ucl.ac.uk
}

\begin{abstract}
The applications of Total Variation (TV) algorithms for Electrical Impedance Tomography (EIT) have been investigated. The use of the TV regularisation technique helps to preserve discontinuities in reconstruction, such as the boundaries of perturbations and sharp changes in conductivity, which are unintentionally smoothed by traditional $l_{2}$ norm regularisation. However, the non-differentiability of TV regularisation has led to the use of different algorithms. Recent advances in TV algorithms such as Primal Dual Interior Point Method (PDIPM), Linearised Alternating Direction Method of Multipliers (LADMM) and Spilt Bregman (SB) method have all been demonstrated successfully for EIT applications, but no direct comparison of the techniques has been made. Their noise performance, spatial resolution and convergence rate applied to time difference EIT were studied in simulations on 2D cylindrical meshes with different noise levels, 2D cylindrical tank and 3D anatomically head-shaped phantoms containing vegetable material with complex conductivity. LADMM had the fastest calculation speed but worst resolution due to the exclusion of the second-derivative; PDIPM reconstructed the sharpest change in conductivity but with lower contrast; SB had a faster convergence rate than PDIPM and the lowest image error.
\end{abstract}

Keywords: electrical impedance tomography, total variation, regularisation

\section{Introduction}

Electrical Impedance Tomography (EIT) is a method for estimating the internal electrical conductivity distribution of an object by injecting current and measuring voltages using electrodes on the surface (Holder 2004). EIT usually entails the estimation of a large number of discretised conductivity values from a limited number of independent boundary voltage measurements. Therefore, regularisation techniques are necessary to obtain a unique solution from the severely ill-posed EIT problem (Lionheart 2003). These techniques usually introduce prior information such as the differentiable of function (Vauhkonen et al 1998), the statistical distribution of different tissue types (Malone et al 2014) or other known anatomical features, to the inverse problem, and the weight of prior knowledge on the estimated solution can be adjusted by regularisation parameters.

\subsection{Background}


1.1.1. Total variation regularisation technique. Total Variation (TV) is a popular regularisation method which has been applied to a range of imaging modalities (Block et al 2007, Chambolle and Lions 1997, Werlberger et al 2010,) and which can preserve sharp discontinuities in images while removing noise and other unwanted details (Rudin et al 1992). Borsic et al (2010a) demonstrated its potential for EIT image reconstruction, which reconstructed sharp boundaries by using both simulated and in vivo physiological data. The TV's ability to preserve edges in reconstructions is due to its use of $l_{1}$ norm penalty term, which is discontinuous and therefore not differentiable at every point. Consequently, simple gradient-based methods cannot be applied for solving TV regularised problems.

A number of algorithms have been designed in recent years to overcome the non-differentiability of TV and solve it efficiently. For application in EIT, a TV algorithm based on Primal and Dual Interior Point Method (PDIPM) was proposed and was able to reconstruct images using in vivo data. PDIPM converts the non-differentiable optimisation problem to the approximated differentiable formulations by introducing a smoothness parameter and a dual variable using the Cauchy-Schwartz inequality (Borsic et al 2010). The Split Bregman (SB) algorithm was proposed (Goldstein and Osher 2009 ) with generality for solving $l_{1}$ norm regularised problems, based on the concept of Bregman distance from functional analysis. This algorithm has few parameters to adjust and has been shown to have a high convergence rate. Recently, Jung and Yun (2014) presented a first-order TV method, Linearised Alternating Direction Method of Multipliers (LADMM), which improves calculation speed by not computing the second-order derivative, known as the Hessian matrix.

1.1.2. Motivation In theory, all TV algorithms should produce the same solution as they are processing the same question (Chambolle 2004, Chambolle and Pock 2010, Wu and Tai 2010). Nevertheless, in practice, the performance of the different TV algorithms varies widely for specific applications because they have different approximations and adjusting parameters. Furthermore, even though it has been proven that the SB method is equivalent to the Alternating Direction Method of Multipliers (ADMM) (Wu and Tai 2010), which is the prototype of LADMM, but with the secondorder derivative included, it is worthwhile comparing LADMM and SB, as the exclusion of the second-order derivative can change the performance of algorithms dramatically.

The above algorithms all hold potential for reconstructing EIT images, with successful simulated and experimental results, but so far there has been no systematic comparison of these TV algorithms, particularly for applications on large-scale models with millions of elements, commonly used for EIT of brain function. Consequently, a comparison study of these TV algorithms would be useful to aid their applications in EIT.

\subsection{Purpose}

The overall purpose of this study was to evaluate the performance of different TV algorithms for EIT. In this study, these algorithms were applied to 2D simulations, cylindrical phantom and a head-shaped phantom experiment, using large meshes. Their performance was compared and their potential for use in time-difference EIT of brain function was assessed. The questions addressed were as follows:

(1) Which algorithm gives the best results?

(2) What are the recommendations for future use in brain EIT?

\subsection{Experimental design}

Images were reconstructed using three TV regularisation algorithms: PDIPM, SB and LADMM, and the first-order Tikhonov algorithm, a traditional quadratic reconstruction algorithm, which was used as a reference. This algorithm, sometimes referred to as the Laplace filter algorithm, also measures the total amplitude of the differentiable but with $l_{2}$ norm, and is suitable for comparison with TV algorithms to show the preservation of edges. In order to assess the performances of TV algorithms in 
practical geometries, as used in EIT of brain function, phantoms of increasing complexity were used. Firstly, a 2D cylindrical mesh was used for simulations with different noise levels, and then experiments were carried out on a cylindrical tank and an anatomically realistic head-shaped phantom. Experimental data was collected using the UCLH Mark 2.5 system (McEwan et al 2006), and the simulations were set to match the specifications of this hardware. This system, based on one module of the Sheffield Mk3.5 system, has a wide frequency range from $20 \mathrm{~Hz}$ to $1.6 \mathrm{MHz}$, with a multiplexer that can address up to 64 electrodes. All the tests were undertaken in linear time difference conditions, and with zero conductivities as the initial guess under room temperature. All considered meshes were quality-checked with the Joe-Liu quality measure (Liu and Joe 1994) with the quality parameter being $>0.9$ for $99.99 \%$ elements.

\section{Methods}

\subsection{General considerations}

2.1.1. Forward problem. The forward problem requires the determination of the boundary voltages for a given object with known conductivity and can be solved using the finite element method (FEM) (Calderón 2006). Considering an imaging body $\Omega$ with a sufficiently smooth boundary $\partial \Omega$ and conductivity $\sigma$, we have scalar potential $\phi$ and the electric field $\mathbf{E}=-\nabla \phi$. Through the continuum version of Ohm' law and continuum Kirchoff's law, the following partial differential equation is obtained (Holder 2004):

$$
\nabla \cdot \sigma \nabla \phi=0 \text { in } \Omega
$$

The boundary conditions are introduced by modelling electrodes according to the complete electrode model (Somersalo et al 1992). Supposing the subset of the boundary in contact with the electrode is $E_{l}$, we have the boundary with electrodes $\Gamma=\bigcup_{l} E_{l}$ and without electrodes $\Gamma^{\prime}=\partial \Omega-\Gamma$. The complete electrode model is shown:

$$
\begin{gathered}
\phi+z_{l} \gamma \frac{\partial \phi}{\partial \mathbf{n}}=0 \text { on } \Gamma \\
\int_{E_{l}} \gamma \frac{\partial \phi}{\partial \mathbf{n}}=I_{l} \\
\frac{\partial \phi}{\partial \mathbf{n}}=0 \text { on } \Gamma^{\prime}
\end{gathered}
$$

2.1.2. Inverse problem. The goal of the inverse problem is to estimate the internal conductivity distribution by fitting the boundary voltage measurement. The simplest approach is to minimise the sum of squares error.

$$
\|\mathbf{V}-F(\boldsymbol{\sigma})\|^{2}
$$

where $\mathbf{V}$ denotes the measured boundary voltages, $\boldsymbol{\sigma}$ is the conductivity and $F$ represents the forward operator. Usually the norm is a standard $l_{2}$ norm. Prior information, which also can be treated as penalty term, can be added:

$$
\min _{\boldsymbol{\sigma}} \mathrm{f}(\boldsymbol{\sigma})=\|\mathbf{V}-F(\boldsymbol{\sigma})\|^{2}+G(\boldsymbol{\sigma})
$$

A standard regularisation is:

$$
G(\boldsymbol{\sigma})=\lambda\left\|\mathbf{L}\left(\boldsymbol{\sigma}-\boldsymbol{\sigma}_{r e f}\right)\right\|^{2}
$$

where $\mathbf{L}$, the regularisation matrix, is commonly an identity matrix, partial differentiable matrix and diagonal matrix; $\lambda$ denotes the regularisation parameter. The minimisation problem becomes a tradeoff between the fidelity term and regularisation term. 
2.1.3. Total variation regularisation. The $l_{2}$ norm is commonly used in regularisation term due to its differentiability, but the solution of the minimisation problem (2) will be biased towards smoother functions, for which the $l_{2}$ norm assumes has a smaller value. The total variation regularisation technique still uses the differentiable regularisation matrix but with a $l_{1}$ norm measurement, which does not penalise image discontinuities. Consequently, the TV regularisation technique is particularly suited to reconstructing sharp changes. For a differentiable function on a domain $\Omega$, the total variation is ( Chan and Wong 1998, Dobson and Vogel 1997, Holder 2004, Rudin et al 1992, Vogel and Oman 1996 ):

$$
T V(\mathbf{f})=\int_{\Omega}|\nabla \mathbf{f}|
$$

\subsection{TV algorithms}

2.2.1. PDIPM. An efficient method for solving the non-differentiable TV regularised problem of EIT has been proposed, which is based on the primal-dual theory developed by Andersen et al (1999).

The original TV regularised inverse problem is non-differentiable, so it is modified by the introduction of a smoothness parameter:

$$
T V(\boldsymbol{\sigma})=\int_{\Omega} \sqrt{|\nabla \boldsymbol{\sigma}|^{2}+\beta}
$$

where $\beta>0$ denotes the smoothness parameter. The origin minimisation problem with the above TV regularisation term is labelled as the primal problem. A new dual variable is introduced to the primal problem according to the Cauchy-Schwartz inequality.

$$
\max _{\chi_{i}: \chi_{i} \mid \leq 1} \min _{\boldsymbol{\sigma}}\|\mathbf{V}-\mathrm{F}(\boldsymbol{\sigma})\|^{2}+\lambda \sum_{i} \chi_{i} \mathbf{L}_{i} \boldsymbol{\sigma}
$$

where $\chi$ represent the dual variable. The above maximisation problem is labelled as the dual problem, and the primal and dual problems have the same optimum solution. Therefore, the optimal point can null the gap between the two problems:

$$
\left|\mathbf{L}_{i} \boldsymbol{\sigma}\right|-\chi_{i} \mathbf{L}_{i} \boldsymbol{\sigma}=0 i=1, \ldots, n .
$$

which is called the complementarity condition.

The multi-variable Gauss Newton method can be used to solve the PDIPM problem. The updates of primal and dual variables are given by:

$$
\begin{gathered}
\delta \boldsymbol{\sigma}=-\left[\mathbf{J}^{T} \mathbf{J}+\lambda \mathbf{L}^{T} \mathbf{E}^{-1} \mathbf{K} \mathbf{L}\right]^{-1} \cdot\left[\mathbf{J}^{T}(\mathbf{V}-\mathrm{F}(\boldsymbol{\sigma}))+\lambda \mathbf{L}^{T} \mathbf{E}^{-1} \mathbf{L} \boldsymbol{\sigma}\right] \\
\mathbf{E}=\operatorname{diag}\left(\sqrt{\left|\mathbf{L}_{i} \boldsymbol{\sigma}\right|^{2}+\beta}\right) \mathbf{K}=\operatorname{diag}\left(1-\frac{\boldsymbol{\chi}_{i} \mathbf{L}_{i} \boldsymbol{\sigma}}{\sqrt{\left|\mathbf{L}_{i} \boldsymbol{\sigma}\right|^{2}+\beta}}\right) \\
\delta \boldsymbol{\chi}=-\boldsymbol{\chi}+\mathbf{E}^{-1} \mathbf{L} \boldsymbol{\sigma}+\mathbf{E}^{-1} \mathbf{K} \mathbf{L} \delta \boldsymbol{\sigma}
\end{gathered}
$$

where $\mathbf{J}$ denotes the Jacobian matrix.

The primal problem more closely approximates to the original TV regularised problem if the smoothness parameter $\beta$ is small. Too small a value of $\beta$ will causes divergence since the TV regularised problem is non-differentiable. In this paper, the initial value used for this parameter was 1e-4, to match the default value given in EIDORs (Adler and Lionheart 2006), and decreased for each iteration.

2.2.2. $S B$ method. It is first necessary to define the concept of "Bregman Distance", before we describe the SB method. The Bregman Distance associated with a convex function $E$ at the point $\mathbf{v}$ is:

$$
D_{E}^{\mathbf{p}}(\mathbf{u}, \mathbf{v})=E(\mathbf{u})-E(\mathbf{v})-<\mathbf{p}, \mathbf{u}-\mathbf{v}>
$$

Where $\mathbf{p}$ is in the subgradient of $E$ at $\mathbf{v}$. 
We define $H(\boldsymbol{\sigma})=\|\mathbf{V}-\mathrm{F}(\boldsymbol{\sigma})\|^{2}$ and introduce a variable $\mathbf{d}=\mathrm{L}(\boldsymbol{\sigma})$. The inverse problem with regularisation can be written as:

$$
\min _{\mathbf{\sigma}, \mathbf{d}}|\mathbf{d}|+H(\boldsymbol{\sigma}) \text { subject to } \mathbf{d}=\mathrm{L}(\boldsymbol{\sigma})
$$

If we denote $E(\boldsymbol{\sigma}, \mathbf{d})=|\mathbf{d}|+H(\boldsymbol{\sigma})$, the above unconstrained problem can be recast by introducing a Largangian function and inserting the Bregman distance (Goldstein and Osher 2009).

$$
\begin{gathered}
\left(\boldsymbol{\sigma}^{k+1}, \mathbf{d}^{k+1}\right)=\min _{\mathbf{u}, \mathbf{d}}|\mathbf{d}|+H(\boldsymbol{\sigma})+\frac{\lambda}{2}\left\|\mathbf{d}-\mathrm{L}(\boldsymbol{\sigma})-\mathbf{b}^{k}\right\|_{2}^{2} \\
\mathbf{b}^{k+1}=\mathbf{b}^{k}+\left(\mathrm{L}\left(\boldsymbol{\sigma}^{k+1}\right)-\mathbf{d}^{k+1}\right)
\end{gathered}
$$

It is necessary to decouple the two variables in the (11a), since the computation of (11b) is trivial. The updates of $\boldsymbol{\sigma}$ and $\mathbf{d}$ can be calculated separately after splitting the $l_{1}$ norm and $l_{2}$ norm parts.

$$
\begin{aligned}
& \text { Step1 } \boldsymbol{\sigma}^{k+1}=\min _{\boldsymbol{\sigma}} H(\boldsymbol{\sigma})+\frac{\lambda}{2}\left\|\mathbf{d}^{k}-\mathrm{L}(\boldsymbol{\sigma})-\mathbf{b}^{k}\right\|_{2}^{2} \\
& \text { Step2 } \mathbf{d}^{k+1}=\min _{\mathbf{d}}|\mathbf{d}|+\frac{\lambda}{2}\left\|\mathbf{d}^{k}-\mathrm{L}\left(\boldsymbol{\sigma}^{k+1}\right)-\mathbf{b}^{k}\right\|_{2}^{2}
\end{aligned}
$$

2.2.3. $L A D M M$. ADMM has been proposed to resolve nondifferentibility in minimisation problem (12). This method overcomes the difficulty by introducing splitting scheme and soft thresholding. The augmented Lagrangian function is applied in ADMM to convert (12) into an unconstrained problem. The augmented Lagrangian function is used to convert (12) into an unconstrained problem.

$$
F_{\alpha}(\boldsymbol{\sigma}, \mathbf{d}, \mathbf{p}):=H(\boldsymbol{\sigma})+\lambda|\mathbf{d}|+<\mathbf{p}, \mathbf{d}-L(\boldsymbol{\sigma})>+\frac{\alpha}{2}\|\mathbf{d}-L(\boldsymbol{\sigma})\|_{2}^{2}
$$

where $\mathbf{p}$ denotes the new introduced variable, $\lambda$ and $\alpha$ are the parameters. The function in (16) is linearised and the proximal term $\frac{1}{2 \delta}\|\boldsymbol{\sigma}-\tilde{\boldsymbol{\sigma}}\|^{2}$ is added. The linearised augmented Lagrangian function is produced:

$$
F F_{\alpha}(\boldsymbol{\sigma}, \mathbf{d}, \mathbf{p}, \tilde{\boldsymbol{\sigma}}, \delta):=<\nabla H(\boldsymbol{\sigma})+\alpha\left(\mathbf{d}-L(\boldsymbol{\sigma}), \boldsymbol{\sigma}-\tilde{\boldsymbol{\sigma}}>+\frac{1}{2 \delta}\|\boldsymbol{\sigma}-\tilde{\boldsymbol{\sigma}}\|^{2}+\lambda|\mathbf{d}|+<\mathbf{p}, \mathbf{d}-L(\boldsymbol{\sigma})>\right.
$$

where $\tilde{\boldsymbol{\sigma}}$ denotes the approximation of $\boldsymbol{\sigma}, \delta$ is the parameter. LADMM can be expressed in three phase form after decoupling variables $(\boldsymbol{\sigma}, \mathbf{d}, \mathbf{p})$. The soft thresholding criteria is utilised to further simplify the calculation.

$$
\begin{aligned}
& \boldsymbol{\sigma}^{k+1}=\boldsymbol{\sigma}^{k}-\delta\left(\mathbf{A}^{T}\left(\mathbf{A} \boldsymbol{\sigma}^{k}-\mathbf{b}\right)+\mathbf{L}^{T}\left(\alpha\left(\mathbf{L} \boldsymbol{\sigma}^{k}-\mathbf{d}^{k}\right)-\mathbf{p}^{k}\right)\right) \\
& \mathbf{p}^{k+1}=\mathbf{p}^{k}+\alpha\left(\mathbf{d}^{k+1}-\mathbf{L} \boldsymbol{\sigma}^{k+1}\right) \\
& \mathbf{d}^{k+1}= \begin{cases}\mathbf{L} \boldsymbol{\sigma}^{k+1}-\frac{\mathbf{p}^{k}}{\alpha}-\frac{\lambda}{\alpha} & \text { if } \alpha \mathbf{L} \boldsymbol{\sigma}^{k}-\mathbf{p}^{k} \geq \boldsymbol{\lambda} \\
0 & \text { if }\left|\alpha \mathbf{L} \boldsymbol{\sigma}^{k}-\mathbf{p}^{k}\right| \leq \boldsymbol{\lambda} \\
\mathbf{L} \boldsymbol{\sigma}^{k+1}-\frac{\mathbf{p}^{k}}{\alpha}+\frac{\lambda}{\alpha} & \text { if } \alpha \mathbf{L} \boldsymbol{\sigma}^{k}-\mathbf{p}^{k} \leq-\lambda\end{cases}
\end{aligned}
$$

$\delta=1 /\left\|\mathbf{A}^{T} \mathbf{A}+\alpha \mathbf{L}^{T} \mathbf{L}\right\|$ is suggested (Jung and Yun 2014), but the value, equivalent to the norm of Hessian matrix, is very difficult to calculate for large meshes. Consequently, a group of values were tested and the one with the best image quantification was selected.

2.2.4. Regularisation parameter. To enable comparison between the different TV algorithms, the same regularisation parameter value $\lambda$ was used for all algorithms in each experiment. The value for $\lambda$ was defined heuristically in each experiment by testing 30 values within the range $1 \mathrm{e}-9$ to $1 \mathrm{e}-3$ and 
selecting the value that produced the best results across all algorithms. The L-curve was used for choosing the regularisation parameter for the 1st-order Tikhonov method.

2.2.5. Iteration stop criterion. We note that all three TV methods are iterative, so an iteration terminating criterion based on the relative decrease of the objective functional was adopted.

$$
H(\boldsymbol{\sigma})=\|\mathbf{V}-F(\boldsymbol{\sigma})\|^{2}+\lambda T V(\boldsymbol{\sigma})
$$

The iterative algorithms were stopped when the relative decrease of this functional was smaller than $1 \%$.

$$
\left[\frac{H\left(\boldsymbol{\sigma}_{n+1}\right)}{H\left(\boldsymbol{\sigma}_{n}\right)}-1\right] \leq 0.01
$$

However, the terminating criterion was checked after each 100 iterations for LADMM algorithm because this first-order TV algorithm converges more slowly than the others for each iteration.

\subsection{Experimental setup and process}

2.3.1. Model and phantom. A cylindrical mesh of diameter $19 \mathrm{~cm}$ and height $10 \mathrm{~cm}$, with a ring of 32 electrodes around the centre, was designed, while the boundary voltages were simulated using a mesh with 62784 elements. The ground point was fixed at the centre of the base of the mesh. A current of peak amplitude $133 \mu \mathrm{A}$, injected though polar electrodes, was simulated, and the voltage differences on all adjacent pairs of electrodes not involved in delivering the current were obtained. The electrodes were described with the complete electrode model, and the electrode impedance was set to $1 \mathrm{k} \mathrm{ohm}$. To evaluate the noise performance of the TV algorithms, boundary voltages were simulated with Signal to Noise Ratio (SNR) of $60 \mathrm{~dB}, 40 \mathrm{~dB}$ and $30 \mathrm{~dB}$, generated by adding Gaussian white noise. The conductivity of the background was $0.4 \mathrm{Sm}^{-1}$, to simulate that of the $\mathrm{NaCl}$ solution. A cylindrical perturbation with conductivity of $0.36 \mathrm{Sm}^{-1}$, diameter $4 \mathrm{~cm}$ and height $10 \mathrm{~cm}$ was placed at a point with coordinates (x: $5 \mathrm{~cm} \mathrm{y:} 0 \mathrm{~cm} \mathrm{z:} 0 \mathrm{~cm}$ ), with reference to the centre of the mesh (figure 1).

A Perspex cylindrical tank study was designed to validate the simulations and test the performance of these TV algorithms in 2D. A tank with the same properties as described for the simulations was used. Electrodes were stainless steel discs, $1 \mathrm{~cm}$ in diameter. A current of peak amplitude $133 \mu \mathrm{A}$ and frequency $1 \mathrm{kHz}$ was injected and boundary voltages were measured according to the polar protocol. To mimic the properties of living tissues, biological objects were used as a background. The background medium was a mixture of $0.1 \%$ concentration $\mathrm{NaCl}$ solution and carrot cubes of approximately $4 \mathrm{~mm}$ diameter. The cylindrical potato perturbation of diameter $4.6 \mathrm{~cm}$ and height 10 $\mathrm{cm}$ was placed at $(\mathrm{x}:-4 \mathrm{~cm}$ y: $0 \mathrm{~cm})$ in the saline-carrot mixture (figure 6). The conductivities of the saline-carrot mixture and potato at a frequency of $1 \mathrm{kHz}$ are $0.1 \mathrm{~S} / \mathrm{m}$ and $0.02 \mathrm{~S} / \mathrm{m}$ respectively.

We designed an anatomical head-shaped phantom to test these algorithms in 3D condition. 32 sliver electrodes and a ground electrode were positioned based on the distribution proposed by (Avery 2014). Electrodes were addressed using the protocol eeg $31 \mathrm{~b}$, in which current is preferentially applied to diametrically opposing electrodes (Tidswell et al 2001). The same potato perturbation was placed in posterior (x: $4.5 \mathrm{~cm}$ y: $0 \mathrm{~cm} \mathrm{z:} 0 \mathrm{~cm})$, middle $(\mathrm{x}: 8 \mathrm{~cm}$ y: $0 \mathrm{~cm} \mathrm{z:} 0 \mathrm{~cm})$ and anterior $(\mathrm{x}: 13 \mathrm{~cm} \mathrm{y:} 0 \mathrm{~cm}$ z: $0 \mathrm{~cm}$ ) positions, with the origin set to the posterior boundary (figure 7). All phantom experiments were undertaken at room temperture.

2.3.2. Conversion of large mesh. A coarse mesh, converted from a fine mesh, was employed for inverse solution to avoid the "inverse crime" and increase the time resolution. A fine mesh of the anatomically realistic head-shaped phantom, with 4 million tetrahedral elements, was used for precalculating the Jacobian matrix and simulating the boundary voltage. A coarse mesh of 79453 hexahedral elements, matching the tetrahedrons of the fine mesh, was used for the inverse problem solution. The Jacobian matrix for the coarse mesh was computed by projecting the Jacobian computed for the fine mesh onto the hexahedral elements. 
2.3.3. Convergence rate. If $\mathrm{m}$ represents the number of measurement and e denotes the number of elements, the most time consuming calculation of the two second-order TV methods is $\mathbf{J}^{T} \mathbf{J}$, whose time complexity for each iteration is $O\left(m \bullet e^{2}\right)$; the corresponding calculation of the LADMM is $\mathbf{J}^{T}(\mathbf{J \sigma})$, whose time complexity for each iteration is $O(2 m \bullet e)$. Thus, it is clear that LADMM is faster than SB and PDIPM in our case since $e \gg 2$. In our experiments the time costs of one iteration of PDIPM and SB, which are very close, are comparable to 1000 iterations of LADMM. Therefore, LADMM is undoubtedly the fastest TV algorithm of those evaluated here. The time cost of PDIPM and SB can be inferred from the number of iteration, as their time consumption of each iteration is similar. The iteration number of convergence is dependent on the applications and stop criterion. The specific computational time of convergence is found through iteration numbers for each experiment.

\subsection{Image quantification}

Image quality was assessed quantitatively by three metrics (Fabrizi et al 2009). Prior knowledge of the perturbation conductivity is required before performing the following three metrics. Pixels are considered part of the perturbation if their conductivities are higher than $50 \%$ of the maximum perturbation conductivity for positive perturbations, or lower than $50 \%$ of the minimum perturbation conductivity for negative perturbations.

Image noise: inverse of the contrast-to-noise ratio between the perturbation $P$ and background $B$

$$
\frac{\operatorname{std}\left(\boldsymbol{\sigma}_{B}\right)}{\mid \overline{\boldsymbol{\sigma}_{P}-\boldsymbol{\sigma}_{B} \mid}}
$$

where $\boldsymbol{\sigma}_{P}$ and $\boldsymbol{\sigma}_{B}$ are the mean intensities of the perturbation and background and std is the standard deviation.

Localisation error: ratio between the norm of the $x-y-z$ displacement of the centre of mass of the reconstructed perturbation $\mathrm{P}$ from the actual position $(x, y, z)$, and the norm of the dimensions of the mesh $\left(d_{x}, d_{y}, d_{z}\right)$. The centre of the mass is found where the weighted relative position of the distributed conductivity sums to zero.

$$
\frac{\left\|\sum_{n \in P}\left(x_{n}, y_{n}, z_{n}\right)-(x, y, z)\right\|}{\left\|\left(d_{x}, d_{y}, d_{z}\right)\right\|}
$$

where $\left(x_{n}, y_{n}, z_{n}\right)$ denotes the position of the centre of the $n$th element.

Shape error: mean ratio of the difference between the dimensions of the simulated and reconstructed perturbations, respectively $\left(l_{x}, l_{y}, l_{z}\right)$ and $\left(l_{x}^{\prime}, l_{y}^{\prime}, l_{z}^{\prime}\right)$, and the dimensions of the mesh. The size of perturbations is found by calculating the difference of the maximal and minimal in each direction.

$$
\frac{1}{3}\left(\frac{\left|l_{x}-l_{x}^{\prime}\right|}{d_{x}}+\frac{\left|l_{y}-l_{y}^{\prime}\right|}{d_{y}}+\frac{\left|l_{z}-l_{z}^{\prime}\right|}{d_{z}}\right)
$$

\section{Results}

\subsection{Noise performance}

All TV algorithms showed good noise immunity in simulations. The boundaries of the perturbation reconstructed by the TV algorithms could still be distinguished, even though the resolutions deteriorated for increasing noise levels. (Image error values: PDIPM 60dB: 0.508, 40dB: 0.519, 30dB: 0.696; SB 60dB: 0.426, 40dB: 0.463, 30dB: 0.612; LADMM 60dB: 0.687, 40dB: 0.721, 30dB: 0.987). It was not possible to precisely locate the internal boundary of the reconstructed images using 1storder Tikhonov regularisation with $30 \mathrm{~dB}$ SNR. The minimum conductivities of perturbations reconstructed by this algorithm were approximately 0.02 to 0.004 for SNR of $60 \mathrm{~dB}$ to $30 \mathrm{~dB}$. TV 
algorithms produced similar conductivity values of perturbations across all noise levels. Artefacts in reconstructed images using PDIPM and SB for lower SNR conditions appeared near the positions of electrodes as the iterations increased. PDIPM reconstructed the most uniform conductivities of background and perturbation, and produced the steepest change in boundaries. The conductivity value using SB was the closest to the real value $-0.04 \mathrm{Sm}^{-1}$ compared to other $\mathrm{TV}$ algorithms. The reconstruction using SB was the only one where the perturbation did not overlap with the boundary of the mesh for $30 \mathrm{~dB}$ of noise, as shown in figure 2(c). LADMM did not reconstruct the uniform conductivities as other second-order TV algorithms and the location of its reconstructed perturbation had a distinct error. Figure 3 demonstrate that the $1^{\text {st }}$ order Tikhonov had the lowest image errors in 60dB SNR and marginally higher errors than SB when noise was increased. LADMM had the highest image errors across all noise levels, and the image noise of PDIPM was larger than the SB.

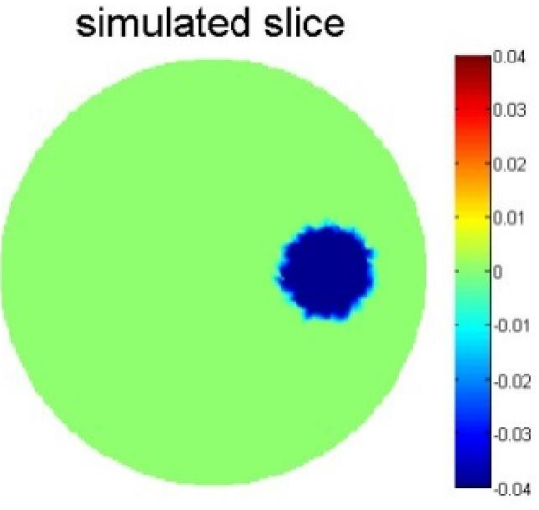

(a)

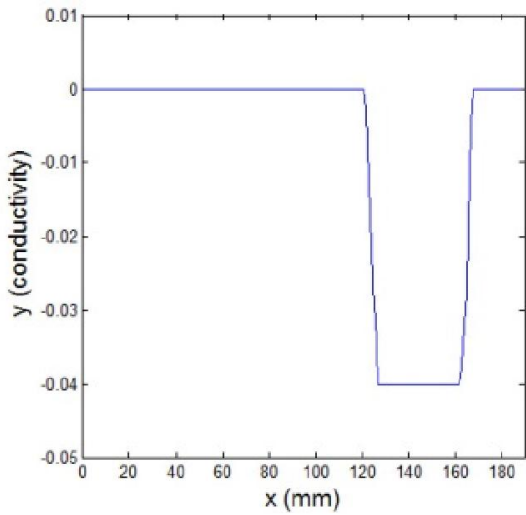

(b)

Figure 1. Simulation model: (a) $2 \mathrm{D}$ slice at $\mathrm{z}=5 \mathrm{~cm}$, (b) profile plots at y $0 \mathrm{~cm}$
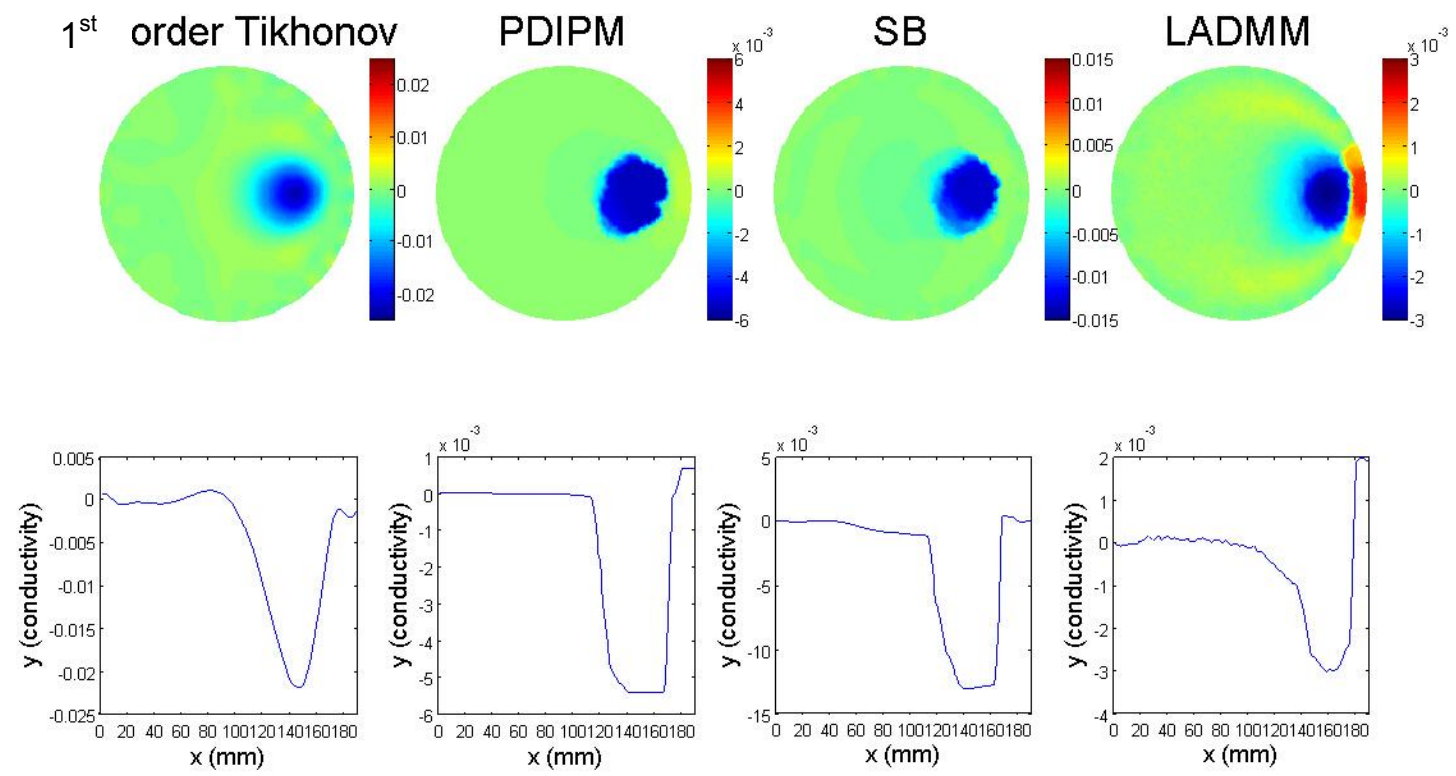

(a) $60 \mathrm{~dB}$ 

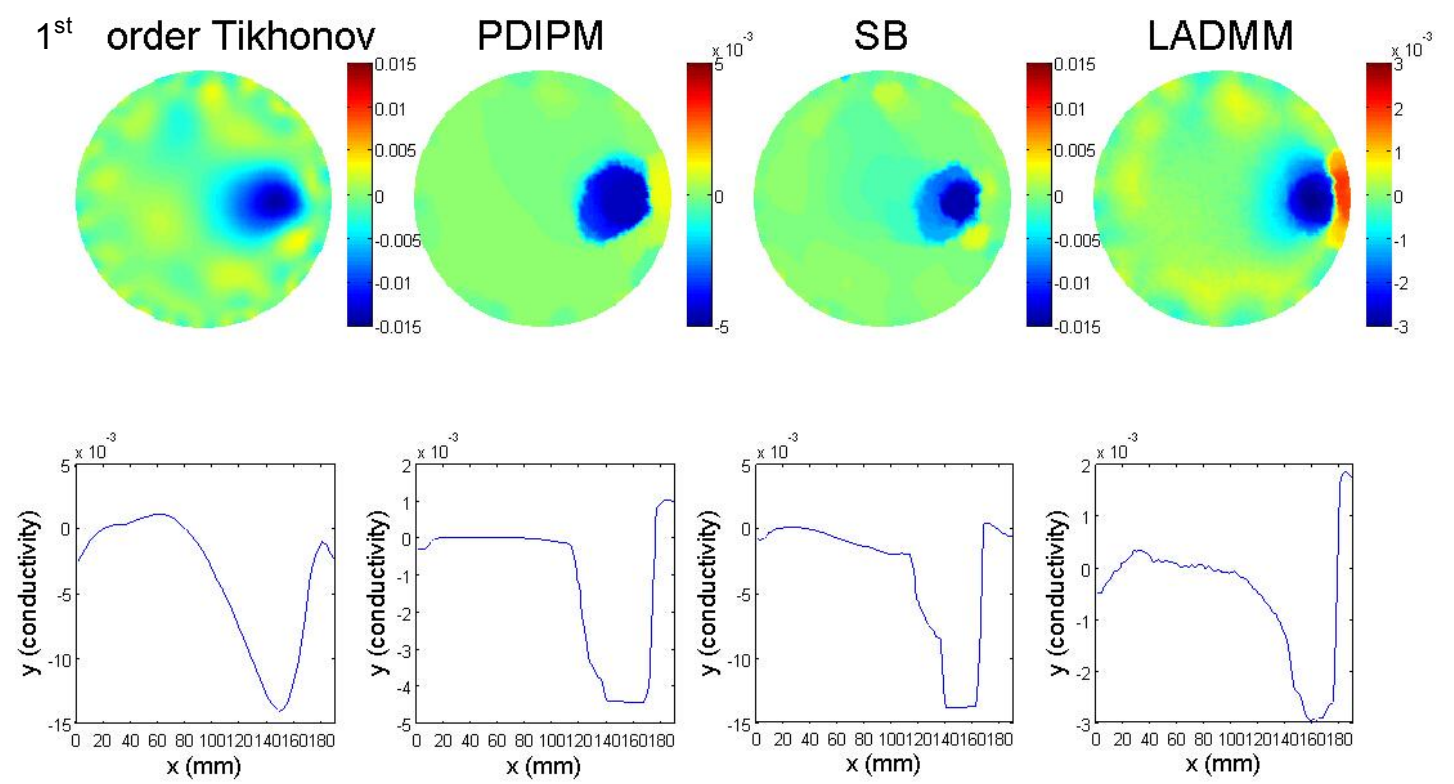

(b) $40 \mathrm{~dB}$
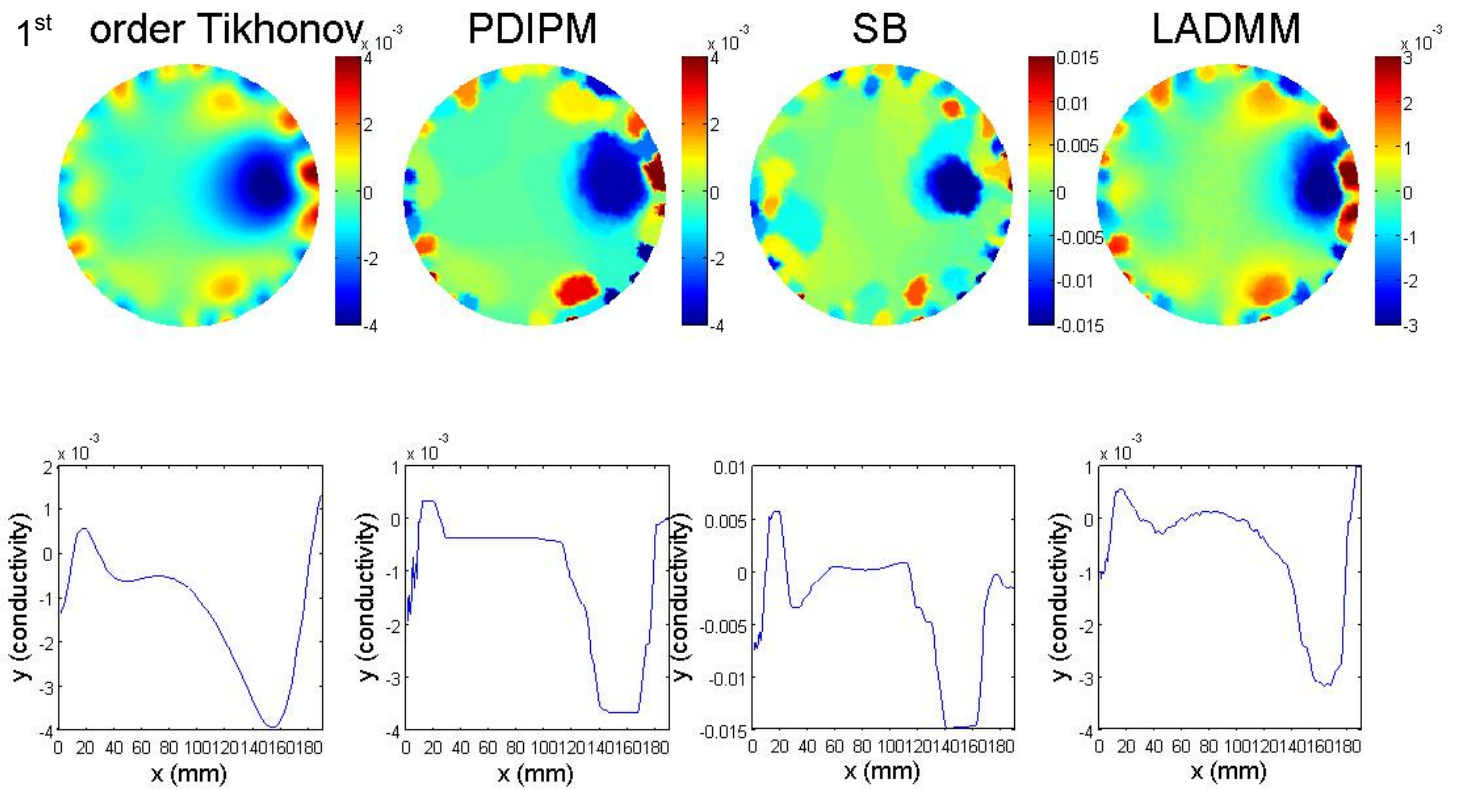

(c) $30 \mathrm{~dB}$

Figure 2. Illustration of the noise performance of the algorithms: 2D slice at $\mathrm{z}=5 \mathrm{~cm}$ and profile at y 0 cm of (a) $60 \mathrm{~dB} \mathrm{SNR}$, (b) $40 \mathrm{~dB} \mathrm{SNR}$, (c) $30 \mathrm{~dB}$ SNR 


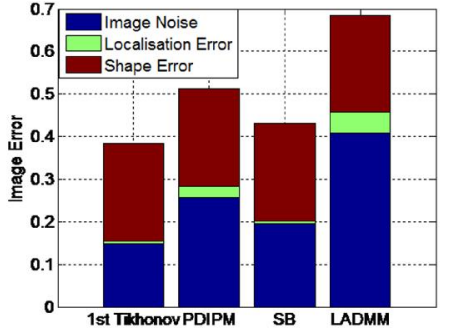

(a) $60 \mathrm{~dB}$

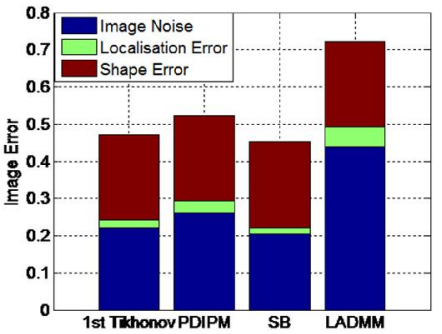

(b) $40 \mathrm{~dB}$

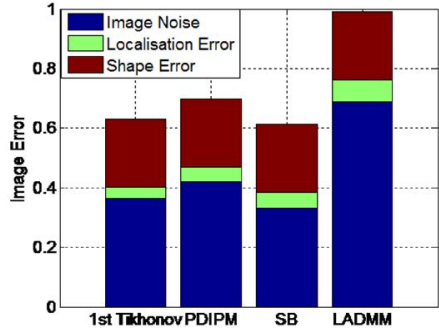

(c) $30 \mathrm{~dB}$

Figure 3. Image error of (a) $60 \mathrm{~dB}$ SNR, (b) $40 \mathrm{~dB}$ SNR, (c) $30 \mathrm{~dB}$ SNR

\subsection{Iteration time}

SB converged faster than PDIPM (figure 4). PDIPM and SB both needed more iterations to converge as the noise increased, and fewer iterations of SB were required than PDIPM in all simulations. LADMM quickly produced the overall characteristics and sharpened the edge slowly after several hundred iterations. The image error curve of PDIPM reconstruction became flat after 2 iterations with $30 \mathrm{~dB}$ SNR, while the curve of SB descended until the $7^{\text {th }}$ iteration.

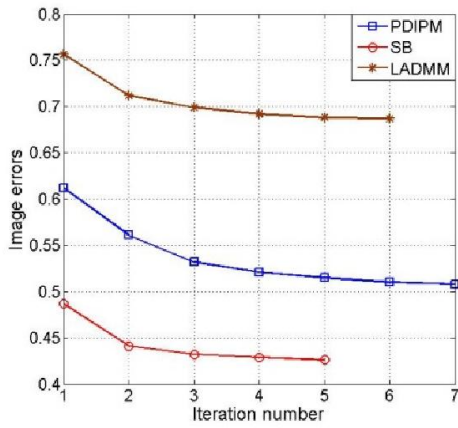

(a) $60 \mathrm{~dB}$

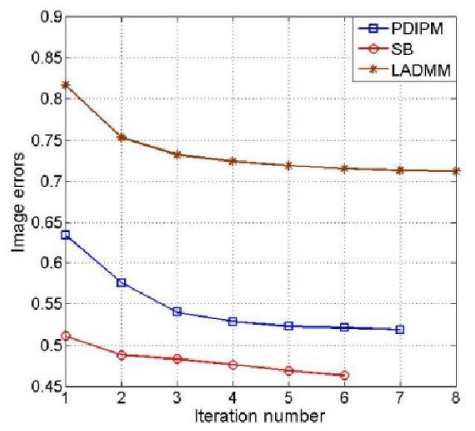

(b) $40 \mathrm{~dB}$

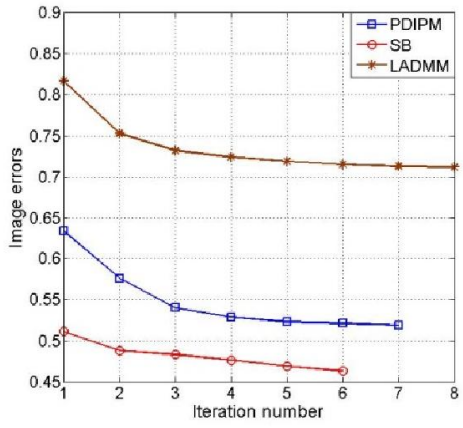

(c) $30 \mathrm{~dB}$

Figure 4. (a) Convergence performance of $60 \mathrm{~dB}$ SNR, (b) convergence performance of $40 \mathrm{~dB}$ SNR, (c) convergence performance of $30 \mathrm{~dB}$ SNR

\subsection{Cylinder tank results}

All four algorithms reconstructed the position of the potato perturbation. PDIPM had the most uniform reconstruction which can be seen in profiles of figure 5. However, the area of the reconstructed perturbation of this TV algorithm was larger than the actual perturbation in figure 6(a). The shape and conductivity value of the reconstruction of SB were more accurate compared to PIDPM, even though the interior boundary was not as steep. There was a clear difference between the boundary of perturbation near the centre and the edge for LADMM. It generated severe artefacts in the areas near the centre which can be seen in the slice and profile. The reconstructed perturbation of LADMM was biased towards the surface by around $10 \mathrm{~mm}$, as seen in the simulations. The iteration numbers for SB and PDIPM were 5 and 6, while LADMM used 700 iterations to convergent. 


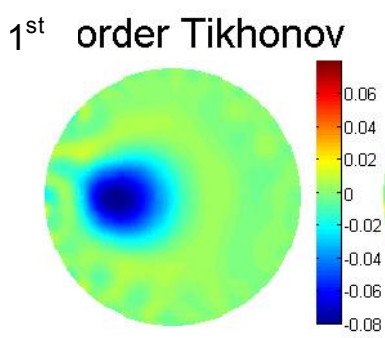

\section{PDIPM}

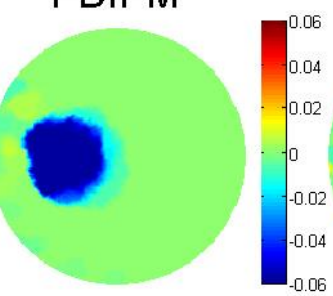

SB

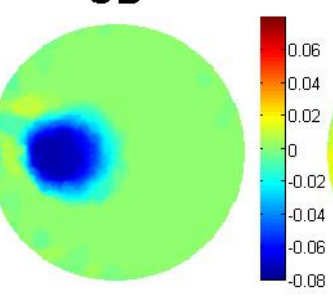

\section{LADMM}

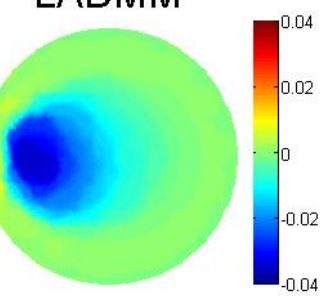

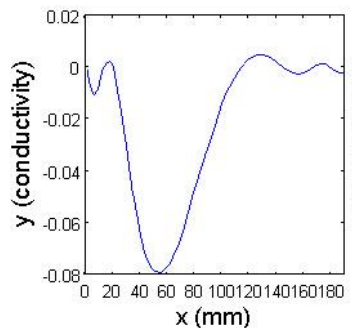
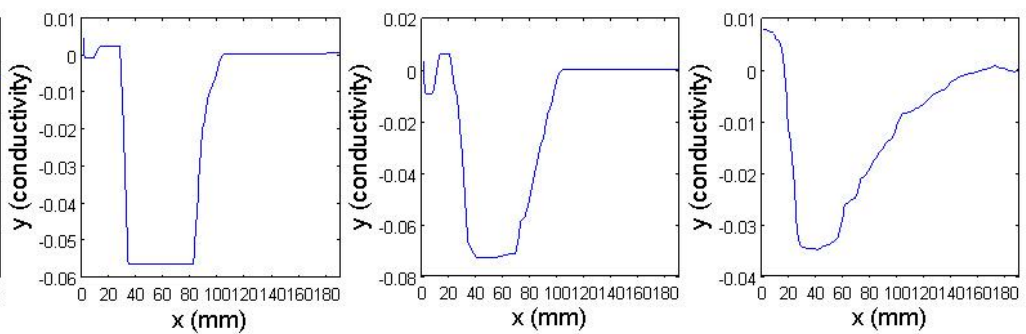

Figure 5. $2 \mathrm{D}$ slice at $\mathrm{z}=5 \mathrm{~cm}$ and profile plots at $\mathrm{y}=0 \mathrm{~cm}$ of the four algorithms for the cylindrical tank experiment

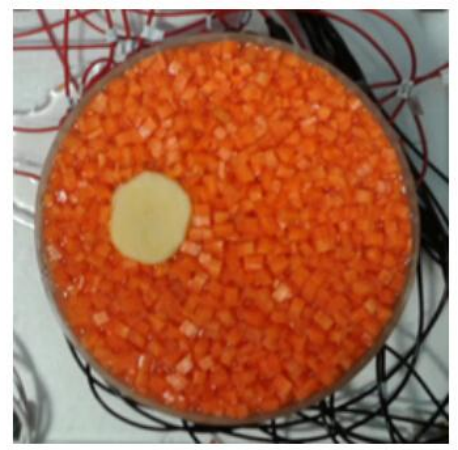

(a)

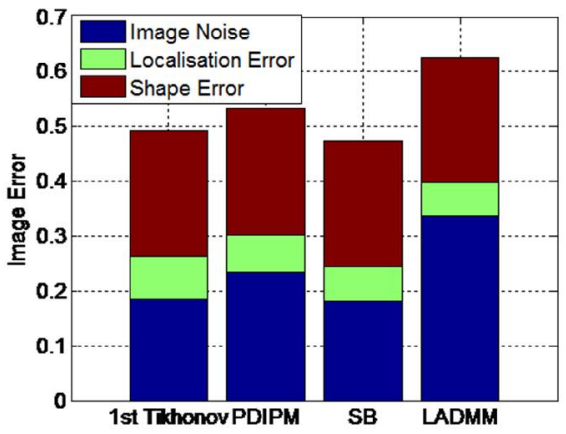

(b)

Figure 6. (a) Cylindrical tank experiment setup, (b) image error of cylindrical tank experiment

\subsection{Head-shaped phantom results}

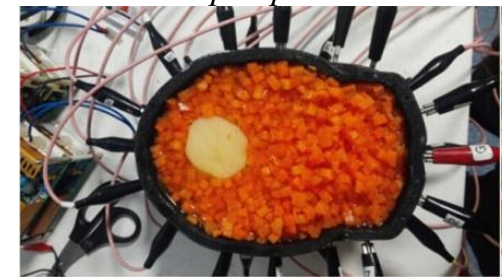

(a) posterior

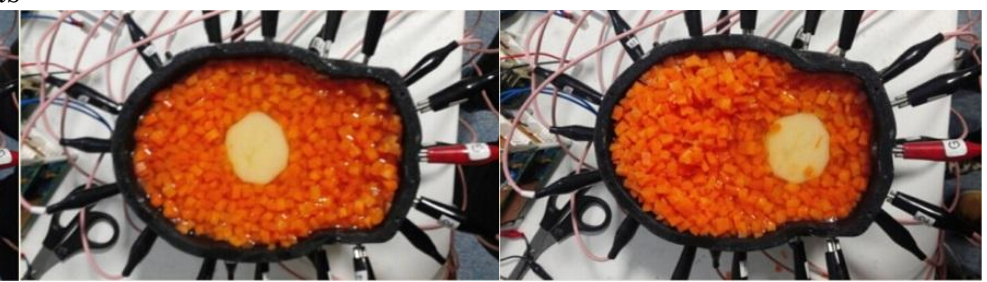

(b) middle (c) anterior

Figure 7. Anatomically head-shaped phantom experiment setup: (a) posterior, (b) middle, (c) anterior

All algorithms could reconstruct the images for three different perturbation locations, although the images obtained for the posterior position were the most accurate (figure 8). The smoothest reconstructed images were obtained by the $1^{\text {st }}$ order Tikhonov algorithm, with large artefacts in background. The PDIPM and SB reconstructions showed a greater sharpness for the localised conductivity change and a greater uniformity for the background and perturbation. The reconstructed 
images in figure 8 show that the reconstructed perturbation of LADMM is enlarged and that the position was biased towards the edge of the phantom. The shapes of reconstructed perturbations obtained using PDIPM and SB were clearly distorted towards a square. In terms of image errors, figure 9 illustrates that SB had the lowest image errors while the image errors of LADMM were the highest. The localisation error of LADMM for all experiments was worse than other algorithms. The image noise of PDIPM was larger than SB, matching the lower contrast of the reconstructions of PDIPM. In terms of iteration numbers, SB employed 8, 11 and 9 iterations to find the optimum in posterior, middle and anterior placement, fewer than $8,13,10$ iterations used by PDIPM. The iteration numbers of LADMM were 1100, 1700, 1400.
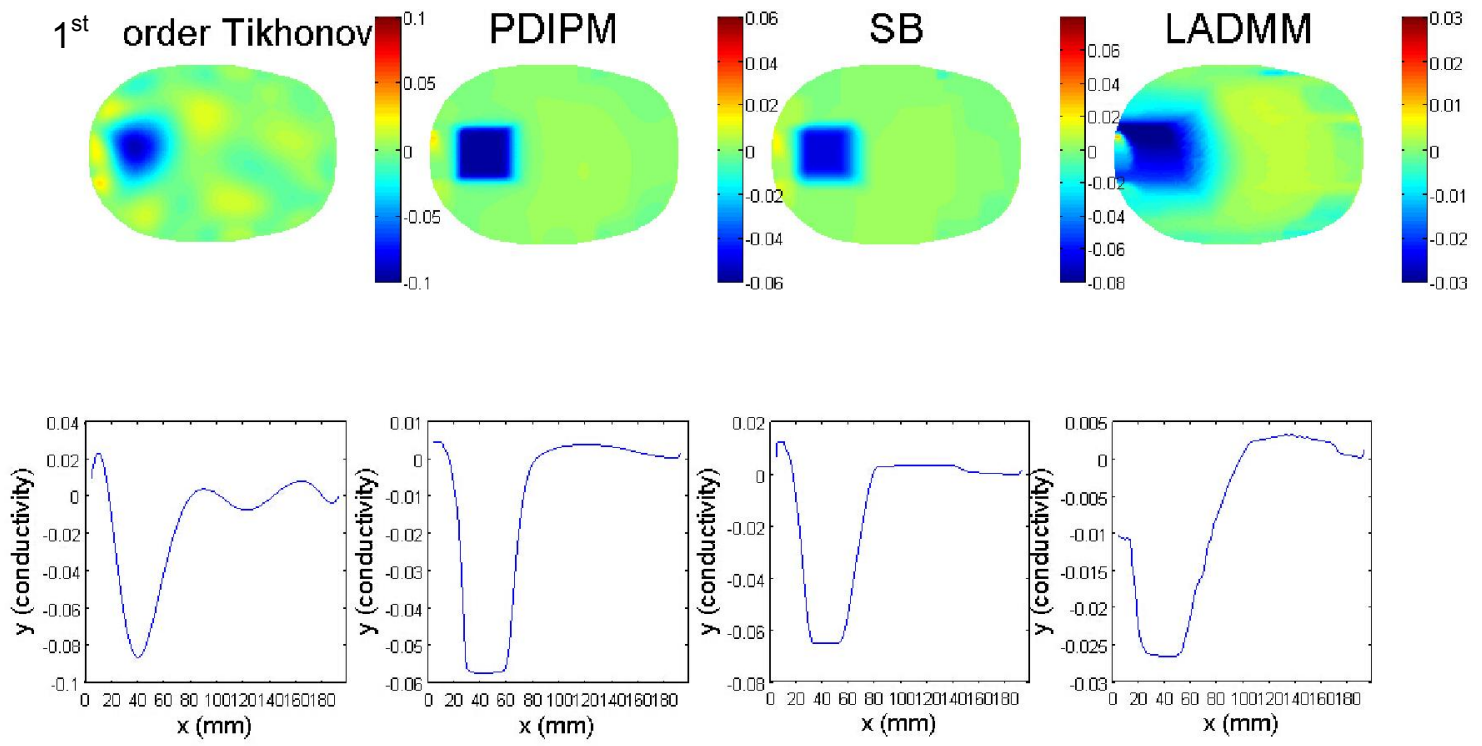

(a) posterior
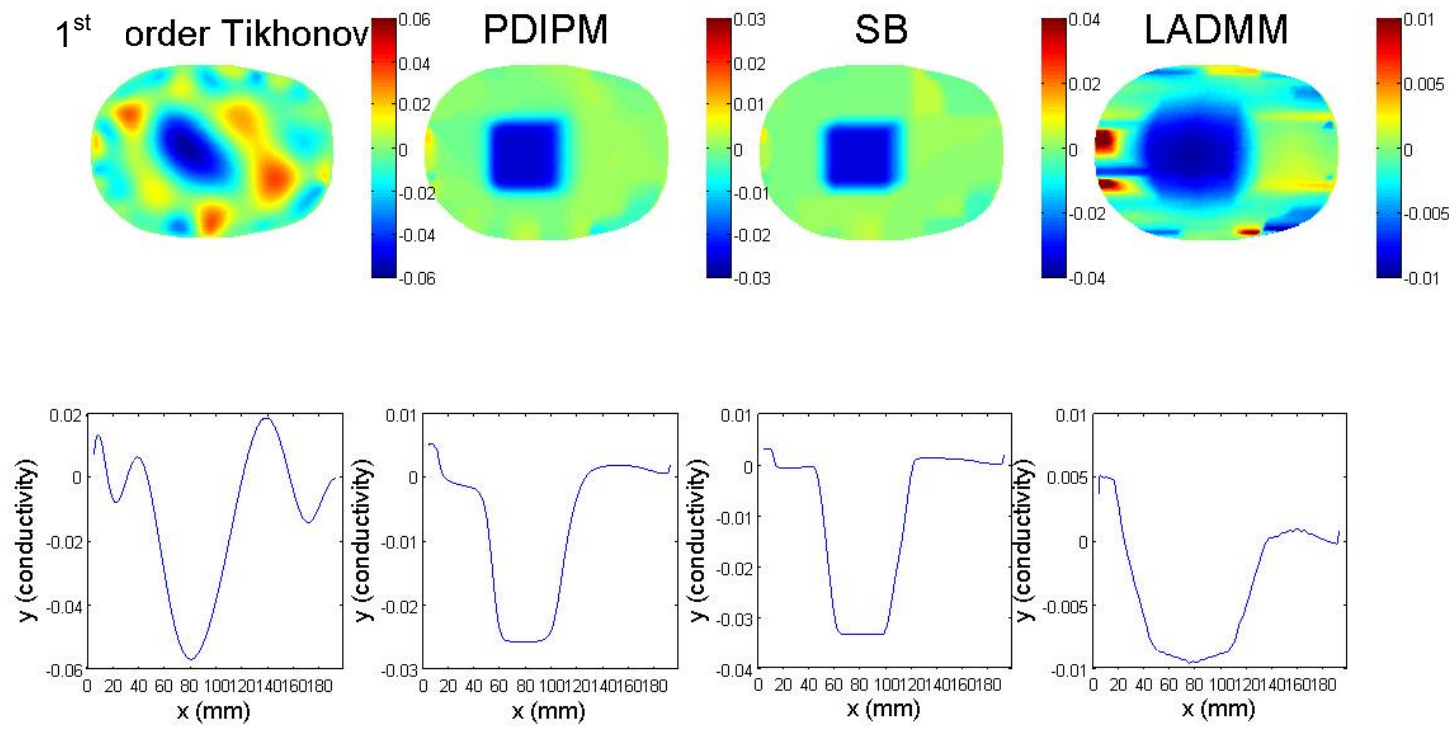

(b) middle 

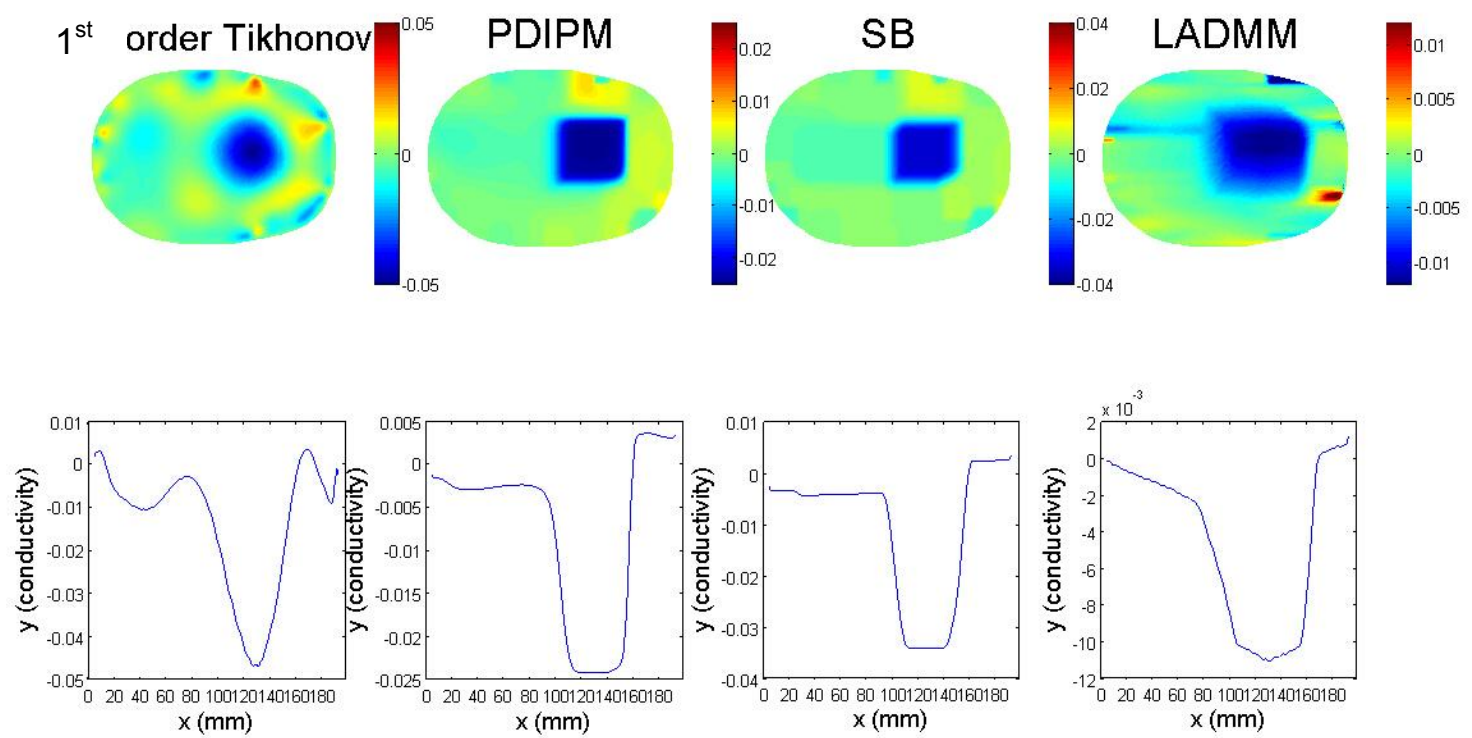

(c) anterior

Figure 8. Reconstruction of the three perturbation positions in the head-shaped tank for each algorithm: $2 \mathrm{D}$ slice at $\mathrm{z}=7 \mathrm{~cm}$ and profile at $\mathrm{z}=0 \mathrm{~cm}$ of (a) posterior perturbation, (b) middle perturbation, (c) anterior perturbation

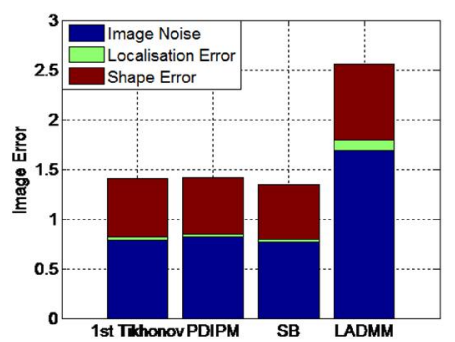

(a) posterior

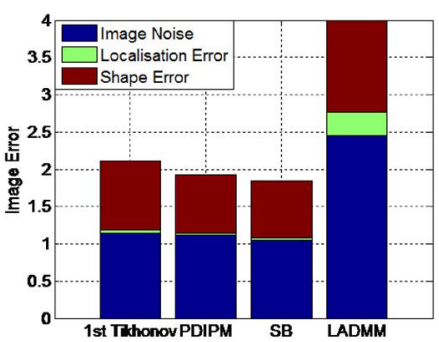

(b) middle

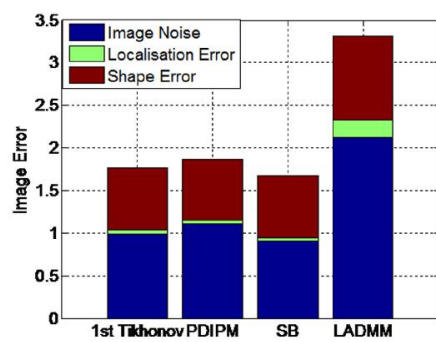

(c) anterior

Figure 9. (a) Image error of posterior perturbation, (b) image error of middle perturbation, (c) image error of anterior perturbation

\section{Discussion}

\subsection{Summary of results}

The image reconstructions revealed significant difference between these TV algorithms for all the experiments.

(1) The TV class algorithms were superior to $1^{\text {st }}$ order Tikhonov algorithm with respect to noise immunity as they reconstructed similar conductivity values across all noise levels. PDIPM and SB demonstrated better artefact resistance with SNR of 40dB. Of the TV algorithms, SB showed the best noise immunity. The reconstructed perturbation of PDIPM and LADMM enlarged as SNR was decreased, whereas SB reconstructed a similar shape for all noise levels.

(2) LADMM produced an approximate reconstruction rapidly and later decreased the image error slowly. The iterations of SB were fewer than PDIPM but this advantage disappeared when the noise was high.

(3) With regards to the spatial resolution of the reconstructions, SB and PDIPM produced similar conductivity distributions and image errors, and furthermore the images of PDIPM reconstructed sharper edges of perturbations with lower contrast than SB; the $1^{\text {st }}$ order 
Tikhonov method reconstructed EIT images better than LADMM but worse than the other two TV algorithms. The cylindrical experiment revealed that PDIPM was able to reconstruct the most uniform image but enlarged the perturbation. LADMM cannot reconstruct the parts of perturbation near the centre from the head-shaped phantom experiment.

\subsection{Technical issues}

The parameters of these algorithms were selected by a heuristic method, and could be improved by using a more accurate algorithm, such as cross validation. The iteration stop criterion was implemented, but in principle the best results would be obtained by removing any limit on the computation time. The diameter and location of the perturbations in the head-shaped phantom experiment were not precise, and the image quality of anterior placement was poorer than posterior placement. This may have been because of the arrangement of electrodes and the eeg31 protocol, which may be addressed by optimising the measurement protocol.

Selection of the optimal parameters for TV algorithms is a difficult task which has not yet been solved in the literature. Addressing this was outside the scope of this work. Thus, the parameters were therefore chosen heuristically (Borsic et al 2010, Jung and Yun 2014) and made the same for all cases ( Jung and Yun 2014).

In this study, we undertook experimental work with the simplified case of an anatomically realistic tank without the skull, as this presents a lower bound for the first evaluation of the comparison of these TV approaches. In future studies, we plan to re-evaluate the methods of the more demanding case of a tank containing a skull, as SNR is lower.

\subsection{Assessment of the TV algorithms}

With regard to the image error, SB method is the best, mainly due to its more accurate conductivity value reconstruction. PDIPM can reconstruct the sharpest change and gives the flattest interior of perturbation and background but with lower contrast conductivities than SB. Initial, rough reconstructions can be obtained by LADMM, but it cannot converge to the optimum solution and always has a position bias.

The TV algorithms have better noise resistance than the first-order Tikhonov algorithm, which is evident in the difference in spatial resolution. The reconstructions of perturbation are quite similar for LADMM in all noise conditions, and are more stable than SB and PDIPM. We suppose that this is because the first-order algorithm avoids the calculation of the approximated Hessian matrix, which may be more likely to be contaminated by noise. SB can reconstruct similar conductivities of perturbation across all noise levels compared to the 10 times change of the first-order Tikhonov algorithm, shown in figure 2 .

In term of calculation time, LADMM is undoubtedly the fastest algorithm due to the exclusion of the second-order derivative. When comparing only the second-order derivative algorithms, the SB method converges faster than PDIPM. Nevertheless, the two algorithms have similar time consumptions when SNR is low.

We suppose that the advantage of the SB method over PDIPM is because the latter introduces the smoothness parameter. The larger the smoothness parameter, the greater the efficiency will be at the expense of accuracy. Consequently, the selection of smoothness parameter become a trade-off between the accuracy and the efficiency ( $\mathrm{Wu}$ and Tai 2010) and only can be processed using a heuristic method.

As the mesh size increases, the number of unknown conductivities becomes larger, but the amount of information obtained through measurements remains the same, which means the ill-posedness of the inverse problem becomes more severe. Due to the lack of information included in second-order derivative, the spatial resolution of LADMM deteriorates as the problem become more ill-posed.

The resolution and noise resistivity are more of a concern for brain EIT, since the inclusion of skull and scalp make the reconstruction more difficult. The SB method recommended for EIT of brain 
function owing to its high resolution, noise resistivity and relatively fast convergence rate, when compared to PDIPM. In terms of other EIT applications, LADMM may be a promising choice for some lung and breast applications requiring a fast algorithm, especially when small mesh sizes are used. For other applications, the SB method may be a suitable choice for its stability and relatively high spatial resolution.

\subsection{Recommendations for future work}

The TV algorithms, different than conventional $l_{2}$ norm regularised algorithms, usually have more than one parameters to be selected, which induce more computational time and hardware. This drawback has become one signification bottleneck for applying the TV methods on EIT, so the parameter selection methods of the TV algorithms will be investigated. ADMM is an alternative TV method. It was not studied in this work because it has been shown to be equivalent to SB in math. However, it might have practical advantages and it may be valuable to compare this method in any future studies.

\section{References}

Adler A and Lionheart W 2006 Uses and abuses of EIDORS: An extensible software base for EIT Physiol. Meas. 27 S25-42 Online: http://iopscience.iop.org/0967-3334/27/5/S03

Andersen K D, Christiansen E, Conn A R and Overton M L 1999 An Efficient Primal-Dual InteriorPoint Method for Minimizing a Sum of Euclidean Norms SIAM J. Sci. Comput. Online: http://citeseerx.ist.psu.edu/viewdoc/summary?doi=10.1.1.46.555

Avery J 2014 Improving Electrical Impedance Tomography of brain function with a novel servocontrolled electrode helmet. Unpublished PhD thesis. University College London.

Block K T, Uecker M and Frahm J 2007 Undersampled radial MRI with multiple coils. Iterative image reconstruction using a total variation constraint. Magn. Reson. Med. 57 1086-98 Online: http://www.ncbi.nlm.nih.gov/pubmed/17534903

Borsic A, Graham B M, Adler A and Lionheart W R B 2010 In vivo impedance imaging with total variation regularization. IEEE Trans. Med. Imaging 29 44-54 Online: http://www.ncbi.nlm.nih.gov/pubmed/20051330

Calderón A P 2006 On an inverse boundary value problem Comput. \&amp; Appl. Math. 25 133-8 Online: http://www.scielo.br/scielo.php?script=sci_arttext\&pid=S180703022006000200002\&lng=en\&nrm=iso\&tlng=en

Chambolle A 2004 An Algorithm for Total Variation Minimization and Applications J. Math. Imaging Vis. 20 89-97

Chambolle A and Lions P-L 1997 Image recovery via total variation minimization and related problems Numer. Math. 76 167-88 Online: http://link.springer.com/10.1007/s002110050258

Chambolle A and Pock T 2010 A First-Order Primal-Dual Algorithm for Convex Problems with Applications to Imaging J. Math. Imaging Vis. 40 120-45 Online: http://link.springer.com/10.1007/s10851-010-0251-1 
Chan T F and Wong C K 1998 Total variation blind deconvolution. IEEE Trans. Image Process. 7 370-5 Online: http://www.ncbi.nlm.nih.gov/pubmed/18276257

Dobson D C and Vogel C R 1997 Convergence of an Iterative Method for Total Variation Denoising SIAM J. Numer. Anal. 34 1779-91 Online: http://epubs.siam.org/doi/abs/10.1137/S003614299528701X

Fabrizi L, McEwan A, Oh T, Woo E J and Holder D S 2009 An electrode addressing protocol for imaging brain function with electrical impedance tomography using a 16-channel semi-parallel system. Physiol. Meas. 30 S85-101 Online: http://iopscience.iop.org/0967-3334/30/6/S06

Goldstein T and Osher S 2009 The Split Bregman Method for L1-Regularized Problems SIAM J. Imaging Sci. 2 323-43 Online: http://epubs.siam.org/doi/abs/10.1137/080725891

Holder D S 2004 Electrical Impedance Tomography: Methods, History and Applications. IOP Publishing, Bristol, UK. Online: http://books.google.com/books?hl=en\&lr=\&id=cjcRd4m_jUQC\&pgis=1

Jung Y M and Yun S 2014 Impedance Imaging with First Order TV Regularization. IEEE Trans. Med. Imaging Online:

http://www.researchgate.net/publication/265094550_Impedance_Imaging_with_First_Order_TV _Regularization

Lionheart W R B 2003 EIT Reconstruction Algorithms: Pitfalls, Challenges and Recent Developments Physiol. Meas. Online: http://arxiv.org/abs/physics/0310151

Liu A and Joe B 1994 Relationship between tetrahedron shape measures BIT 34 268-87 Online: http://link.springer.com/10.1007/BF01955874

Malone E, Sato Dos Santos G, Holder D and Arridge S 2014 Multifrequency electrical impedance tomography using spectral constraints. IEEE Trans. Med. Imaging 33 340-50 Online: http://www.ncbi.nlm.nih.gov/pubmed/24122550

McEwan A, Romsauerova A, Yerworth R, Horesh L, Bayford R and Holder D 2006 Design and calibration of a compact multi-frequency EIT system for acute stroke imaging. Physiol. Meas. 27 S199-210 Online: http://iopscience.iop.org/0967-3334/27/5/S17

Rudin L I, Osher S and Fatemi E 1992 Nonlinear total variation based noise removal algorithms Phys. D Nonlinear Phenom. 60 259-68 Online:

http://www.sciencedirect.com/science/article/pii/016727899290242F

Somersalo E, Cheney M and Isaacson D 1992 Existence and Uniqueness for Electrode Models for Electric Current Computed Tomography SIAM J. Appl. Math. 52 1023-40 Online: http://epubs.siam.org/doi/abs/10.1137/0152060

Tidswell T, Gibson A, Bayford R H and Holder D S 2001 Three-dimensional electrical impedance tomography of human brain activity. Neuroimage $13283-94$ Online:

http://www.ncbi.nlm.nih.gov/pubmed/11162269 
Vauhkonen M, Vadász D, Karjalainen P A, Somersalo E and Kaipio J P 1998 Tikhonov regularization and prior information in electrical impedance tomography. IEEE Trans. Med. Imaging 17285 93 Online: http://www.ncbi.nlm.nih.gov/pubmed/9688160

Vogel C R and Oman M E 1998 Fast, robust total variation-based reconstruction of noisy, blurred images. IEEE Trans. Image Process. 7 813-24 Online: http://www.ncbi.nlm.nih.gov/pubmed/18276295

Vogel C R and Oman M E 1996 Iterative Methods for Total Variation Denoising SIAM J. Sci. Comput. 17 227-38 Online: http://epubs.siam.org/doi/abs/10.1137/0917016

Werlberger M, Pock T and Bischof H 2010 Motion estimation with non-local total variation regularization 2010 IEEE Computer Society Conference on Computer Vision and Pattern Recognition (IEEE) pp 2464-71 Online:

http://ieeexplore.ieee.org/lpdocs/epic03/wrapper.htm?arnumber=5539945

Wu C and Tai X-C 2010 Augmented Lagrangian Method, Dual Methods, and Split Bregman Iteration for ROF, Vectorial TV, and High Order Models SIAM J. Imaging Sci. 3 300-39 Online: http://dl.acm.org/citation.cfm?id=1958729.1958732 